\title{
Comparison of Radiation Damage in Lead Tungstate Crystals under Pion and Gamma Irradiation
}

\author{
V.A. Batarin ${ }^{\text {a }}$, J. Butler ${ }^{\mathrm{b}}$, T.Y. Chen ${ }^{\mathrm{c}}$, A.M. Davidenko ${ }^{\mathrm{a}}$, \\ A.A. Derevschikov ${ }^{a}$, Y.M. Goncharenko ${ }^{\text {a }}$, V.N. Grishin ${ }^{a}$, \\ V.A. Kachanov ${ }^{a}$, V.Y. Khodyrev ${ }^{\mathrm{a}}$, A.S. Konstantinov ${ }^{\mathrm{a}}$, \\ V.I. Kravtsov ${ }^{a}$, Y. Kubota ${ }^{\text {d, V.S. Lukanin }}{ }^{\text {a }}$, Y.A. Matulenko ${ }^{\text {a }}$, \\ Y.M. Melnick ${ }^{\mathrm{a}}$, A.P. Meschanin ${ }^{\mathrm{a}}$, N.E. Mikhalin ${ }^{\mathrm{a}}$, \\ N.G. Minaev ${ }^{a}$, V.V. Mochalov ${ }^{\mathrm{a}}$, D.A. Morozov ${ }^{\mathrm{a}}$, L.V. Nogach ${ }^{\mathrm{a}}$, \\ A.V. Ryazantsev ${ }^{a}$, P.A. Semenov ${ }^{a}$, V.K. Semenov ${ }^{a}$, \\ K.E. Shestermanov ${ }^{a}$, L.F. Soloviev ${ }^{a}$, S. Stone ${ }^{\mathrm{e}}$, \\ A.V. Uzunian ${ }^{\mathrm{a}, 1}$, A.N. Vasiliev ${ }^{\mathrm{a}}$, A.E. Yakutin ${ }^{\mathrm{a}}$, J. Yarba ${ }^{\mathrm{b}}$ \\ The BTeV electromagnetic calorimeter group \\ ${ }^{\mathrm{a}}$ Institute for High Energy Physics, Protvino, Russia \\ ${ }^{\mathrm{b}}$ Fermilab, Batavia, IL 60510, U.S.A. \\ ${ }^{\mathrm{c}}$ Nanjing University, Nanjing, China \\ ${ }^{\mathrm{d}}$ University of Minnesota, Minneapolis, MN 55455, U.S.A. \\ e Syracuse University, Syracuse, NY 13244-1130, U.S.A.
}

\begin{abstract}
Studies of the radiation hardness of lead tungstate crystals produced by the Bogoroditsk Techno-Chemical Plant in Russia and the Shanghai Institute of Ceramics in China have been carried out at IHEP, Protvino. The crystals were irradiated by a $40 \mathrm{GeV}$ pion beam. After full recovery, the same crystals were irradiated using a ${ }^{137} \mathrm{Cs} \gamma$-ray source. The dose rate profiles along the crystal length were observed to be quite similar. We compare the effects of the two types of radiation on the crystals light output.
\end{abstract}

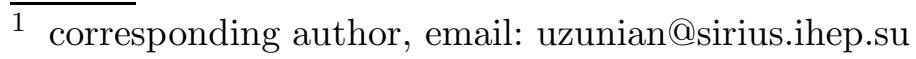




\section{Introduction}

Lead tungstate $\left(\mathrm{PbWO}_{4}\right)$ crystals are planned for use in as the absorptive media in the electromagnetic calorimeters in some collider experiments. The crystals will be irradiated by high energy particles and accumulate significant absorbed doses, up to a several Mrad. The radiation hardness of $\mathrm{PbWO}_{4}$ crystals has been studied by the CMS group using radioactive sources and electron beams [1] and by the BTeV group using both high energy pion and electron beams. A mixture of pion and electron beams provides a particle environment similar to that expected when running the $\mathrm{BTeV}$ experiment $[2,3]$.

The goal of this study is to compare the radiation damage of crystals using two types of irradiation, either a high energy pion beam or a ${ }^{137} \mathrm{Cs}$ radioactive source. If the effects of these two types of radiation are similar, we could use source measurements to classify the radiation resistance of each crystal in the $\mathrm{BTeV}$ like environment. This would be considerably easier than using particle beams for this measurement. Assessing the radiation resistance, is a crucial aspect of crystal quality assurance needed before assembling the electromagnetic calorimeter. However, until now, there has not been a comparative study of $\mathrm{PbWO}_{4}$ crystal radiation damage under hadron beam and gamma source irradiation.

The expected dose distribution in the BTeV crystals is shown in Table 1 for a collider luminosity of $2 \times 10^{32} \mathrm{~cm}^{-2} \mathrm{~s}^{-1}$ and a running time of $10^{7} \mathrm{~s}$.

Table 1

Fraction of $\mathrm{BTeV}$ crystals with given absorbed doses and dose rates estimated at the maximum of the dose profiles inside the crystals $(100 \mathrm{rad}=1 \mathrm{~Gy})$

\begin{tabular}{|c|c|c|}
\hline $\begin{array}{c}\text { Relative } \\
\text { number }(\%)\end{array}$ & $\begin{array}{c}\text { Absorbed dose } \\
(\mathrm{krad} / \text { year })\end{array}$ & $\begin{array}{c}\text { Dose rate } \\
(\mathrm{rad} / \mathrm{h})\end{array}$ \\
\hline \hline 33 & $0.3-2$ & $0.11-0.72$ \\
\hline 27 & $2-5$ & $0.72-1.8$ \\
\hline 12 & $5-10$ & $1.8-3.6$ \\
\hline 16 & $10-50$ & $3.6-18$ \\
\hline 6.2 & $50-100$ & $18-36$ \\
\hline 3.2 & $100-200$ & $36-72$ \\
\hline 2 & $200-500$ & $72-180$ \\
\hline 0.4 & $500-1000$ & $180-360$ \\
\hline 0.2 & $1000-2000$ & $360-720$ \\
\hline
\end{tabular}

Since the BTeV calorimeter starts close to the beam-line, about $15 \mathrm{~cm}$ away, and extends to a radius of $160 \mathrm{~cm}$, the amount of radiation varies greatly. Near the beam-line a small fraction of the crystals, $\sim 1 \%$ have doses of about $10^{4}$ Gy ( 1 Mrad), while $90 \%$ of the crystals have doses less than 0.1 Mrad. The latter corresponds to a dose rate of $36 \mathrm{rad} / \mathrm{h}$. 
About $70 \%$ of the crystals have dose rates $<4 \mathrm{rad} / \mathrm{h}$.

We used three crystals produced by the Bogoroditsk Techno-Chemical Plant in Russia (denoted as CMS-2442, CMS-2443, Bogo-2313) and three more crystals produced by the Shanghai Institute of Ceramics in China (denoted as Shan-T16, Shan-T19, Shan-T20 ). All the crystals except those which are marked as CMS were produced at the end of 2000. The CMS crystals were produced in 2001. The dimensions of the crystals were $\sim 27 \times 27$ $\mathrm{mm}^{2}$ in cross section and $220 \mathrm{~mm}$ in length. The CMS crystals had the same length but were tapered; the dimension at the back is $29 \times 29 \mathrm{~mm}^{2}$. Each crystal was wrapped using $170 \mu \mathrm{m}$ thick Tyvek.

The crystals were irradiated by a $40 \mathrm{GeV} \pi^{-}$beam, four of them in April 2001 and the other two, the CMS crystals, in April 2002. After the recovery process at room temperature the crystals were irradiated by ${ }^{137} \mathrm{Cs} \gamma$-source in May and June 2003. We provided very similar irradiation conditions, in terms of dose rates and absorbed doses, for both cases. Two types of measurements were done on each crystal both before and after the gamma irradiation: a) light output (LO) measurements using a radioactive source for crystal excitation, and b) crystal transparency measurements using a spectrophotometer.

\section{Irradiation by pions}

Pion irradiation was carried out at the IHEP (Protvino) $70 \mathrm{GeV}$ accelerator. The size of the $40 \mathrm{GeV} \pi^{-}$beam was $8 \mathrm{~cm}$ horizontally and $6 \mathrm{~cm}$ vertically, i.e. $90 \%$ of the beam was contained within these dimensions. The beam was present in 1.2 second interval of the full accelerator cycle of 9 sec. Six crystals were irradiated with a dose rate ranging from 30 to $60 \mathrm{rad} / \mathrm{h}$. The crystals light output signals were monitored using the minimum ionizing particles (MIP) in pion beam running and from separate low-intensity calibration runs in electron beam running. LeCroy 2285 15-bit integrating ADC's were used to measure the signal using a $150 \mathrm{~ns}$ integration time. The Protvino testbeam setup is described in detail elsewhere $[3,4,5]$.

\section{Irradiation using a ${ }^{137} \mathrm{Cs}$ source}

We used a radioactive source ${ }^{137} \mathrm{Cs}$, that emits $661 \mathrm{KeV}$ photons and had an activity of $5 \cdot 10^{12} \mathrm{~Bq}$ to irradiate the crystals. The geometrical setup for each crystal position is shown in Fig. 1. Each crystal was irradiated separately. A commercial dosimeter, DKSAT1123, was used to measure the dose rate (in air) in proximity to the crystals. The accuracy is better than $25 \%$ [6].

To modify the spatial dose distribution from the source in order to make it as similar as possible to pion beam, we used a lead screen placed between the source and the crystals 

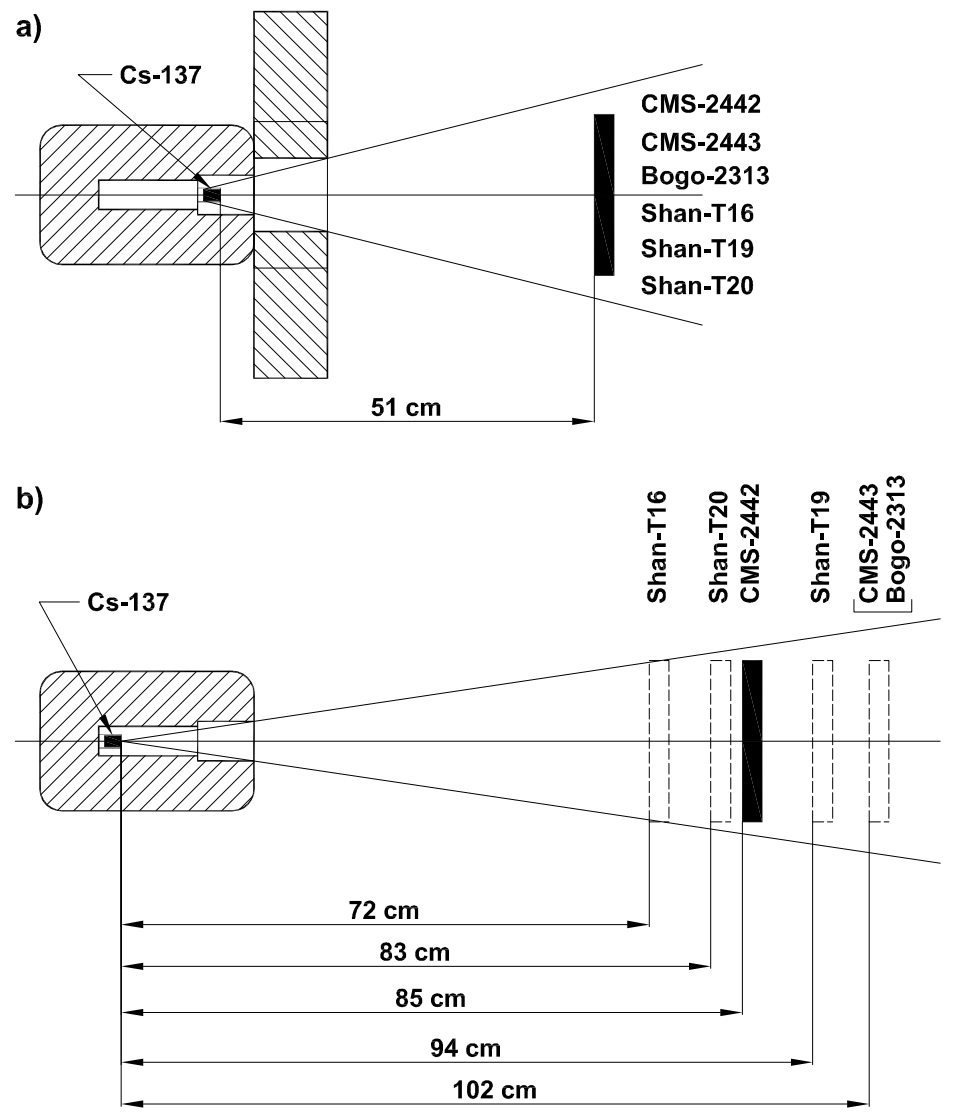

Fig. 1. Setup of the crystal gamma irradiation at the dose rate of $110 \mathrm{rad} / \mathrm{h}(\mathrm{a})$, and the dose rates of $30-60 \mathrm{rad} / \mathrm{h}(\mathrm{b})$.

and also rotated the crystals along their longitudinal axes at a rate of 2 turns per minute. The lead screen was made using four lead plates with varying dimensions [7]. The dose rate distributions along the length of crystal are compared for both the pion and source irradiation in Fig. 2 using the lead screen and rotating the crystals. We see that the profiles are quite similar. The rotation is even more important for insuring a uniform irradiation transverse to the crystal axis when using the source. In Fig. 3 we show the transverse radiation profiles for case (a) where the crystal was not rotated and for case (b) where it was rotated. Since pion irradiation uniformly illuminates the crystals in transverse direction, we use the crystal rotation for all the source results in this paper. The MARS and ROZ-6 codes were used for simulations of the dose profiles in crystal under pion and gamma irradiation respectively $[3,8]$.

Two sets of gamma irradiation were carried out. One of them was done using dose rates of (30-60) rad/h, and the second one at the dose rate of $110 \mathrm{rad} / \mathrm{h}$ for each crystal. The crystals were allowed to recover during two weeks, at room temperature, between the first and the second sets of irradiation. After each exposition the crystal was removed 


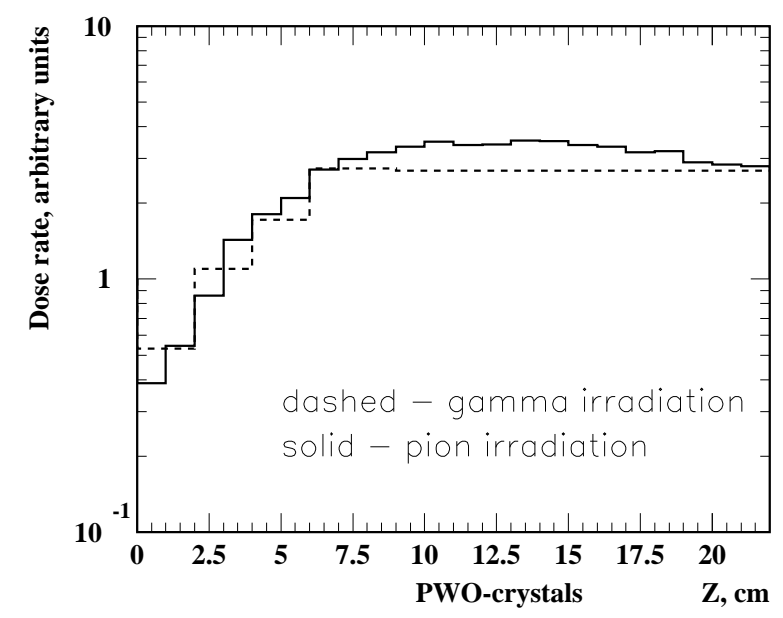

Fig. 2. Longitudinal dose rate profiles for crystal under pion and gamma irradiation(Z near 22 $\mathrm{cm}$ corresponds to the phototube front).
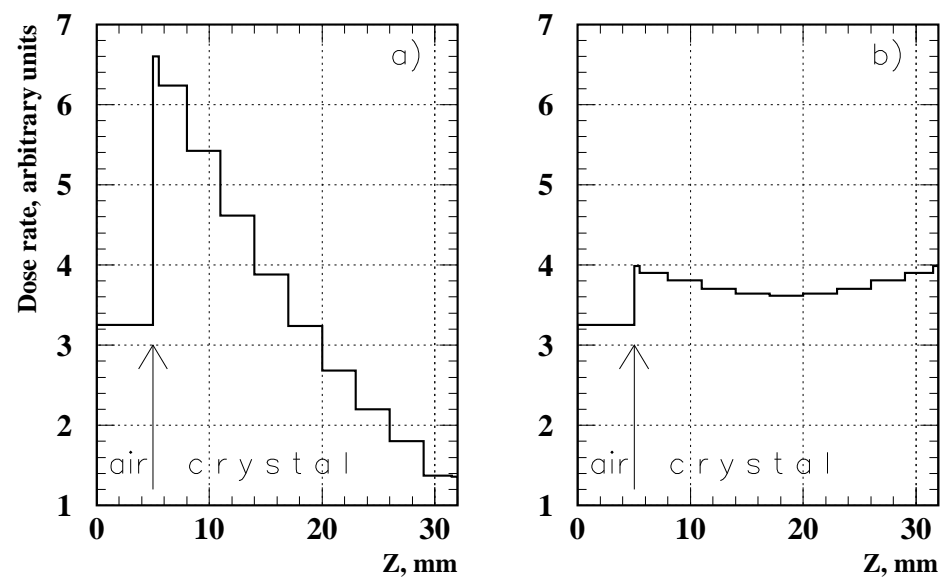

Fig. 3. Transverse dose rate profiles for crystal under ${ }^{137} \mathrm{Cs}$ gamma irradiation: a) side irradiation without crystal rotation, b) side irradiation with crystal rotation. Dose estimations were made in both "air" and "crystal," as indicated.

from the place of irradiation to dedicated test stands for light output and transmittance measurements. This procedure usually took about 1 to 1.5 hours.

\section{Light output and transmittance measurements}

A stand with a radioactive source was used to study changes in the crystal light output due to radiation damage. Scintillation light was detected by a XP2020Q photomultiplier tube (PMT). The PMT output was connected to a $20 \mathrm{kOhm}$ load. The mean current 
through this load was measured by a 11-bit ADC read out by a computer. The PMT high voltage value was of $1.5 \mathrm{kV}$. The PMT nonlinearity in the direct current mode was less than $1 \%$ in the range of $1.2 \mathrm{kV}$ to $2 \mathrm{kV}$. The dark current was $\sim 2 \cdot 10^{-3}$ relative to the average signal level. The background current, mostly due to direct PMT irradiation by the source was $\sim 5 \cdot 10^{-3}$ of the average signal in the worst instance. A ${ }^{137} \mathrm{Cs}$ source $\left(7 \cdot 10^{8} \mathrm{~Bq}\right)$ was installed inside a cylindrical lead collimator, $30 \mathrm{~mm}$ in diameter and 40 $\mathrm{mm}$ in height with aperture diameter of $5.3 \mathrm{~mm}$. The crystals were wrapped by a Tyvek, except for one end which was attached to the PMT without optical grease. The source was moved longitudinally parallel to the central line of the crystal with steps of $2 \mathrm{~cm}$. The distance between the source and the crystal surface was $5 \mathrm{~mm}$. The measurements of the average direct current were provided in the same way for each of the 9 position along the crystal. The mean value over these 9 measurements gives us a value of a light output signal of each crystal. The light output measurements were monitored using a non-irradiated reference crystal. Performing measurements on non-irradiated crystals, we estimate the systematic error to be at most $3 \%$ of the relative light output degradation. The temperature was measured during irradiation and during light output monitoring. The temperature variations were $\sim 1$ degree on average, and up to 2 degrees in the worst case. There were no corrections for the temperature variations. The full systematic errors of the monitoring measurements include the effects of the temperature differences.

To measure the light transmittance of each crystal, a commercial spectrophotometer SF26 was used in the wave length region from 340 up to $700 \mathrm{~nm}$ with a steps of $10 \mathrm{~nm}$. The spectrophotometer light spot was $5 \mathrm{~mm}$ in diameter with a negligible angular dispersion. The measurement accuracy at each point was $1 \%$.

\section{$5 \quad$ Results and discussion}

The dependence of a signal loss on the accumulated dose (at a fixed dose rate) for six crystals irradiated by ${ }^{137} \mathrm{Cs}$ source is shown in Fig. 4. Two crystals lost $10 \%$ of their signal or less under the source irradiation and four other crystals lost (15-25)\%. The light loss in transmittance curves at wavelengths of (420-550) nm measured with the use of the spectrophotometer is one order of magnitude less. For example crystal CMS-2442 showed losses between 2-2.5\% (see Fig. 5). This difference might be due to different optical paths in crystal taken by the injected spectrophotometer light as compared with the scintillation light. From now on we will show only the results from the radioactive source measurements which are more sensitive than the spectrophotometer measurements in showing the effects of radiation.

It has been shown that $\mathrm{PbWO}_{4}$ transmission damage occurs in the crystal when valence electrons are trapped in metastable states around crystal defects. Thus, the irradiation of $\mathrm{PbWO}_{4}$ crystals creates so called color centers which reduce the light attenuation length. When the rate of color centers production (proportional to the dose rate) equals the natural recovery rate, the crystal light output will reach a saturation level [9]. Fig. 4 shows that the signal degrades relatively rapidly with the absorbed dose up to (100-200) rad and then degrades at a significantly slower rate until the saturation level is reached. 

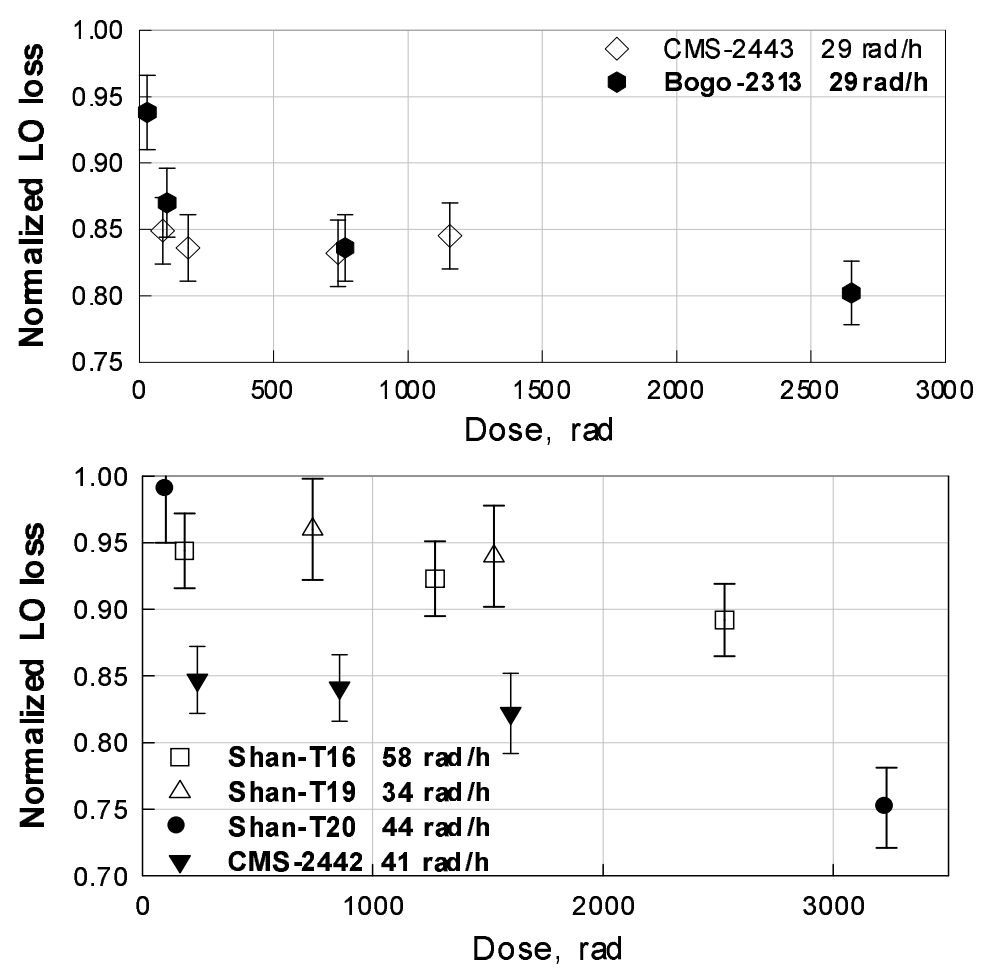

Fig. 4. Light output loss for six crystals versus absorbed dose under gamma irradiation at a fixed dose rate for each crystal. There were several measurements for the two crystals, CMS-2443 and Bogo-2313 (upper part). The results of measurements for other four crystals (bottom part) are consistent with the first two, but were taken at longer intervals.

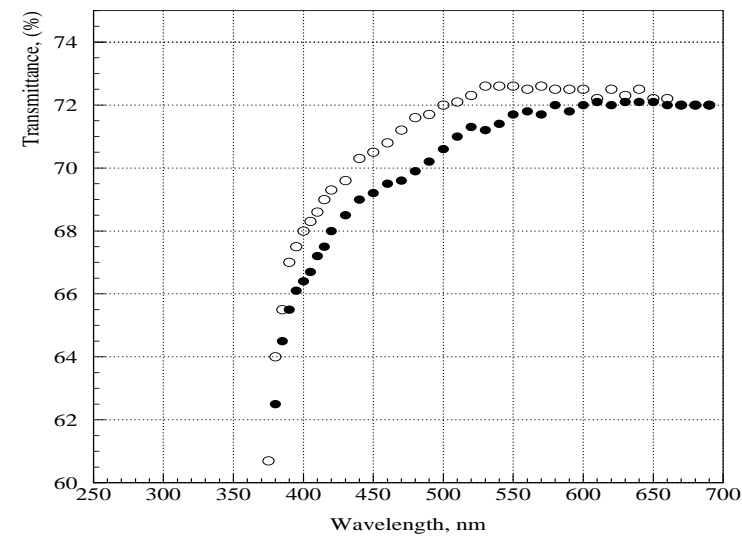

Fig. 5. Transmittance of the CMS-2442 crystal before (open circles) and after (filled circles) gamma irradiation at $41 \mathrm{rad} / \mathrm{h}$ dose rate and $4.4 \mathrm{krad}$ of absorbed dose.

The light loss has a tendency to exhibit saturation when the dose rate is kept at a constant level. Each crystal demonstrates a different level of light loss in the saturated state. We found that the saturation level is reliably reached after $\sim 10$ hours of continuous irradiation at the fixed dose rate. We did not observe a significant difference in radiation hardness of the crystals from different manufacturers.

The second round of gamma irradiation was done at a dose rate of $110 \mathrm{rad} / \mathrm{h}$ for each crystal, after two weeks of recovery at room temperature. Because we irradiated the 


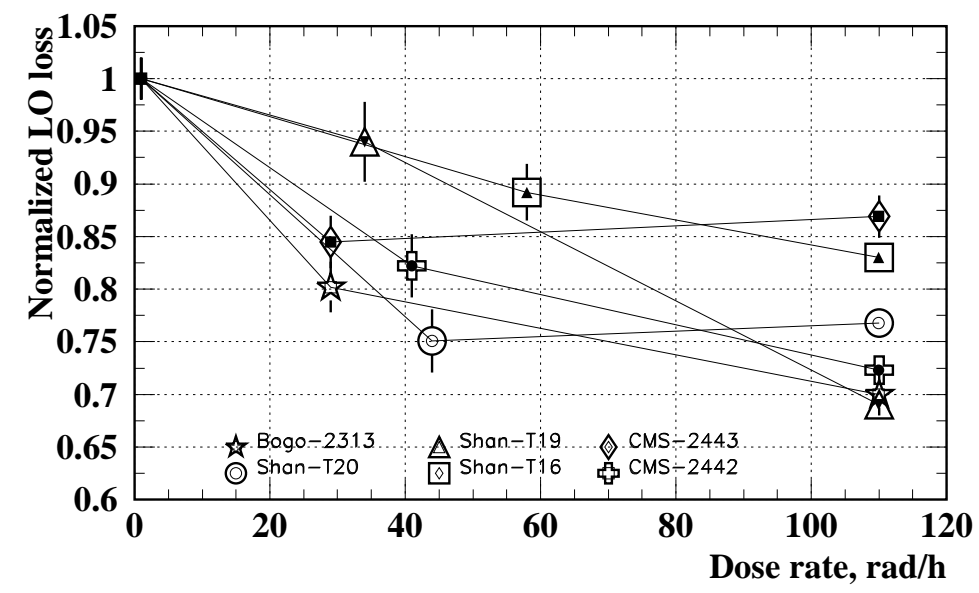

Fig. 6. Dependence of the normalized light output loss (in state of saturation) on a dose rates for six crystals for gamma irradiation using ${ }^{137}$ Cs source.

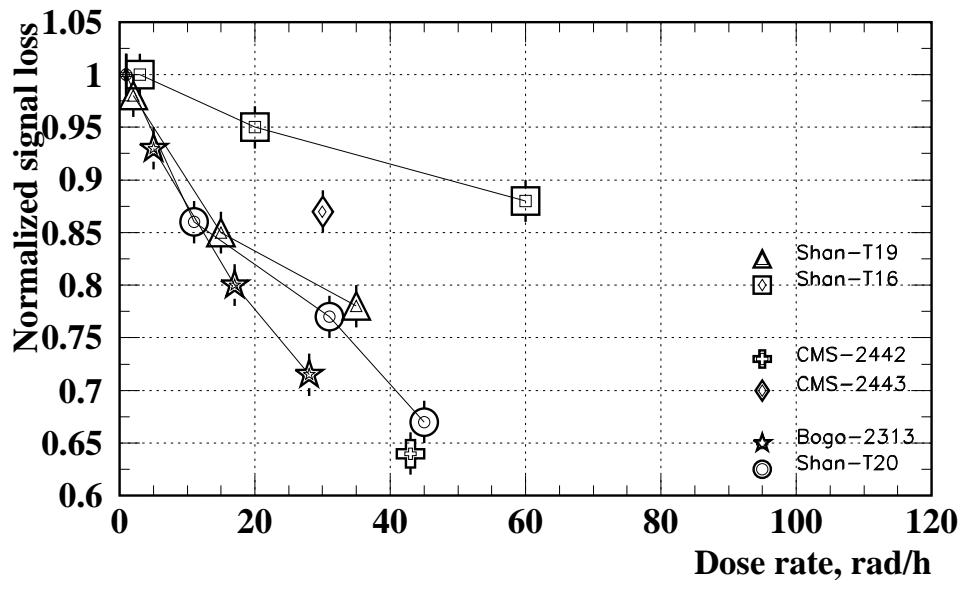

Fig. 7. Dependence of the normalized signal loss (in state of saturation) on a dose rates for 40 $\mathrm{GeV}$ pion irradiation.

crystals with $110 \mathrm{rad} / \mathrm{h}$ gamma-source for a long time, $\sim(60-70)$ hours, we believe that we reached the signal loss saturation level for each crystal at this high dose rate. The dependence of the normalized signal loss (in state of saturation) on the dose rate for the six crystals under gamma irradiation is shown in Fig. 6.

The normalized signal loss versus of the absorbed dose under $40 \mathrm{GeV}$ pion irradiation for the same crystals is presented in Fig. 7. The points on the plot represent the level of signal loss after saturation was reached at the specified fixed dose rates. The rate of the 


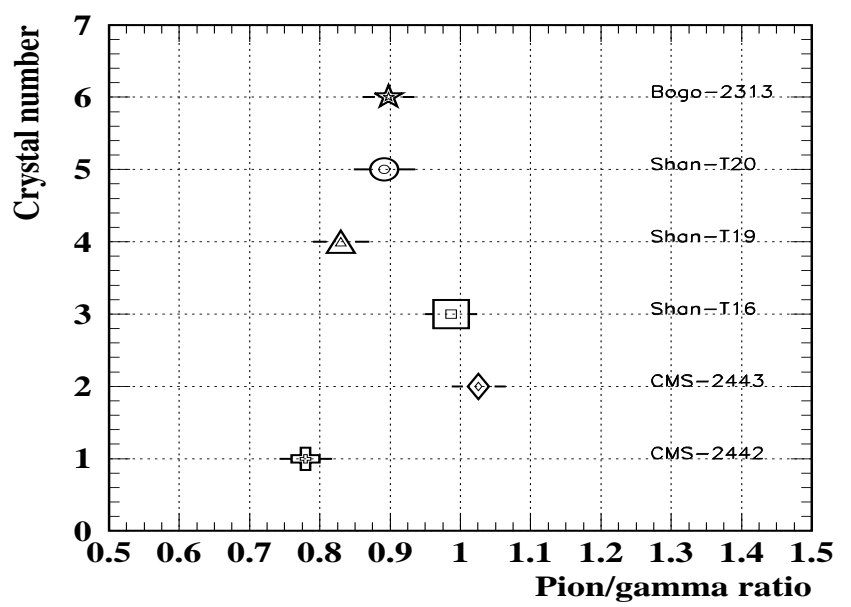

Fig. 8. Ratios of two signal losses at their saturation levels, under pion and under gamma irradiation for the same dose rates $(30-60 \mathrm{rad} / \mathrm{h})$ for six crystals.

signal degradation is practically the same for both irradiation procedures up to the dose rates of $60 \mathrm{rad} / \mathrm{h}$. Using gamma irradiation at a dose rate of $110 \mathrm{rad} / \mathrm{h}$ does not lower the saturation level of some of the crystals, while others are affected (see Fig. 6). This is an indication that the relative difference of the crystal signal losses demonstrated at dose rates of 30-60 rad/h might change at larger dose rates.

Ratios of pion/gamma signal losses at saturation, caused by irradiation at the same dose rates, for the six crystals are shown in Fig. 8. We see that these ratios are close to each other and are in the region between 0.8 and 1 . Thus we can conclude that pion and gamma irradiation at the same dose rates affect lead tungstate crystals in a similar fashion.

We intend to continue our studies; we want to carry out crystal radiation studies for significantly larger numbers of crystals while making continuous light output measurements during the gamma irradiation process.

\section{Summary}

We have observed that the relative signal loss using $40 \mathrm{GeV}$ pion irradiation and using ${ }^{137} \mathrm{Cs}$ radioactive source irradiation looks similar at the dose rate of $(30-60) \mathrm{rad} / \mathrm{h}$.

We have shown that the gamma-source can be used to evaluate the radiation tolerance of $\mathrm{PbWO}_{4}$ crystals. It takes approximately 10 hours of irradiation at fixed dose rates to reach a saturation plateau for each crystal. The dose rate can be chosen in the range of (60-100) $\mathrm{rad} / \mathrm{h}$ because the most of the crystals will be suffering from the lower dose rates at the BTeV experiment. 


\section{Acknowledgments}

This work was partially supported by the U.S. National Science Foundation and the Department of Energy as well as The Russian Foundation for Basic Research grant 02-0239008. We would like to thank the management of IHEP Radiation Research Department for providing us a ${ }^{137} \mathrm{Cs}$ radioactive source for our gamma irradiation studies, as well as the PHENIX group at IHEP (Protvino) for providing us both hardware and software needed for light-output measurements. We thank O. Sumaneev for calculation of the dose profiles in the crystals using gamma irradiation.

\section{References}

[1] CMS, The Electromagnetic Calorimeter Project Technical Design Report, CERN/LHCC 97-33, CMS TDR 4 (1997);

S.Baccaro et al., Further understanding of $\mathrm{PbWO}_{4}$ scintillator characteristics and their optimization, CERN, CMS NOTE 2000/002 (2000);

S. Gascon-Shotkin, Recent Developments in Crystal Calorimeters (featuring the CMS $\mathrm{PbWO}_{4}$ Electromagnetic Calorimeter), CERN, CMS CR 2003/001 (2003);

G.Davies et al., A study of the monitoring of radiation damage to CMS ECAL crystals, performed at X5-GIF,CERN, CMS NOTE 2000/020 (2000);

H.F.Chen et al., Measurements of $\mathrm{PbWO}_{4}$ crystals behavior under irradiation, CERN CMS CR 1999/027 (1999).

[2] A. Uzunian, A. Vasiliev, J. Yarba, First results on simulation of radiation environment at BTeV electromagnetic calorimeter, IHEP preprint 2001-24, Protvino 2001.

[3] V.A.Batarin et al., Study of Radiation Damage in Lead Tungstate Crystals Using Intense High Energy Beams, e-Print ArXiv hep-ex/0210011; Nucl. Instr. and Meth. A 512(2003) 488.

[4] V.A. Batarin et al., Development of a Momentum Determined Electron Beam in the 1-45 GeV Range, e-Print Archive hep-ex/0208012; Nucl. Instr. and Meth. A 510(2003) 211.

[5] V.A. Batarin et al., Precision Measurement of Energy and Position Resolutions of the BTeV Electromagnetic Calorimeter Prototype, e-Print Archive hep-ex/0209055; Nucl. Instr. and Meth. A 510(2003) 248.

[6] http://www.atomtex.com/.

[7] The lead screen was formed of plates with dimensions (thickness, length, height) $\mathrm{xmm}^{3}$ : $19 \times 20 \times 40,12 \times 20 \times 50,7.5 \times 20 \times 50$ and $2 \times 30 \times 50$.

[8] A.V Averin, A.M. Voloschenko, E.P. Kondratenkoet al., The ROZ-6 One-Dimensional Discrete Ordinates Neutrons, Gamma-Rays and Charged Particles Transport Code,

Proc. Int. Topical Meeting on Advaces in Mathematics, Computations and Reactor Physics, Pittsburgh USA, 1991, vol.5.

[9] A.A Annenkov, M.V. Korzhik, P. Lecoq, Lead tungstate scintillation material, Nucl. Instr. and Meth. A 490(2002) 30. 